\title{
NEW SOLID GLASS LASER FOR PHOTODYNAMIC THERAPY
}

\author{
R. GVISHI and R. REISFELD ${ }^{(1)}$ \\ Department of Inorganic and Analytical Chemistry, The Hebrew University of Jerusalem, \\ IL-Jerusalem 91904, Israel
}

\begin{abstract}
A stable tunable solid state laser emitting around $620 \mathrm{~nm}$ is made of a composite glass impregnated by photostable perylimide dye. The laser was pumped with copper vapor laser and the output energy obtained was a few $\mu$ Joul per pulse (repetition rate $50 \mathrm{~Hz}$ ). No significant change in the output power was observed during several hours of operation. Higher output energies are expected. This new laser should be suitable for photodynamic therapy (PDT) and laser-induced fluorescence (LIF) diagnostics in conjunction with hematoporphyrin derivative (HPD) excited at $630 \mathrm{~nm}$.
\end{abstract}

\section{Introduction}

Among the expected advantages of solide state visible laser are medical applications [1], such as photodynamic therapy (PDT) [2-4], which is a new approach for treatment of malignant tumors, and laser-induced fluorescence (LIF) diagnostics $[5,6]$. Both techniques need lasers emitting in the red region of the spectrum. In PDT technique the laser radiation is absorbed by a photosensitizer injected into the patient, which is selectively retained in the malignant tumor cell. The excited photosensitizer molecules transfer energy to the oxygen molecules which are promoted from their ground state ${ }^{3} \Sigma g$ to the excited singlet state ${ }^{1} \Delta g$. Singlet molecular oxygen is known to be a strong cytotoxic agent that violently oxidizes the surrounding (tumor) tissue. Today the common clinicaly used photosensitizer is hematoporphyrin derivative (HPD), known commercially as Photofrin II with two absorption peaks at $405 \mathrm{~nm}$ and $630 \mathrm{~nm}$. The later peak is used for the PDT since the living tissue has a good light transmission at this wavelength. The lasers sources for PDT-HPD, used today, are an Ar-pumped 1iquid dye laser emitting at $630 \mathrm{~nm}$ (output power of milliwatts) and a gold vapor laser emitting at $628 \mathrm{~nm}(50-100 \mathrm{KW}$ peak power with $5-10 \mathrm{~Hz}$ repetition rate and about $30 \mathrm{~ns}$ duration). A more compact and easy to operate smaller system would be advantageous [7].

In LIF a photosensitizer (such as HPD) is concentrated in the tumor and excited. From the shape of its fluorescence spectrum the malignant tumor is detected [5]. A more advanced LIF method does not use the photosensitizer, but detects the pathological changes in the tissue directly from the induced fluorscence spectra of the native tissue [6]. It 's important to note that LIF measurements require much less laser energy than those of PDT. The red radiation of the laser also prevents the photodissociation of the DNA.

Recently $[8,9]$ we succeeded in obtaining a photostable tunable solid state dye laser using a perylimide dye with a laser peak centered at about $575 \mathrm{~nm}$. In this paper we report on a new photostable solid state dye laser (based also on a perylimide dye from BASF) which emits in the red [10] and should be suitable as a source laser for PDT-HPD. Its molecular structure is shown in figure 1.

Enrique Berman Professor of Solar Energy. 
The method by which the dye is incorporated into the glass uses the combination of the sol-gel and organic polymer technique reported earlier [11].

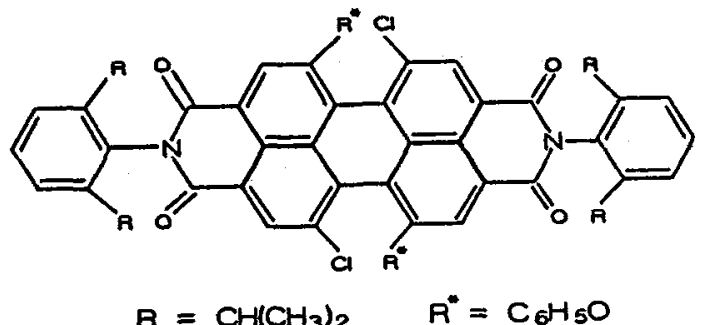

Fig. 1. Structure of perylimide dye used

\section{Experimental}

Spectroscopy: The absorption spectra of the samples were measured on a Lambda 5 Perkin-Elmer double beam spectrophotometer, and fluorescence spectra corrected for the instrumental response were obtained by Jasco type FP770 spectrofluorimeter.

Laser cavity: The dye-doped composite glass was pumped by copper vapor laser (P1asma kinetics) at $510 \mathrm{~nm}$ with $20 \mathrm{~ns}$, full width at half maximum (FWHM) duration, $\sim 2 \mathrm{~mJ} /$ pulse and $\sim 5 \mathrm{KHz}$ repetition rate. The pump beam was chopped to a $\sim 50 \mathrm{~Hz}$ repetition rate and focused to a line (10X0.5 mm) with a combination of spherical and cylindrical lenses. The oscillation cavity consisted of a $100 \%$ reflective metallic back mirror and a $~ 50 \%$ reflective output coupler.

Laser pulse measurement: The laser output spectrum was measured with a $0.25 \mathrm{~m}$ grating monochromator (Oriel model $7240 \mathrm{with} \sim 2 \mathrm{~nm}$ resolution for $0.2 \mathrm{~mm}$ slit) and a Si photodiode power meter (Ophir model PD 2A). The wavelength was scanned manually and several measurements were made at each point. The data presented here are an average of these measurements. The pump pulse power was measured with microprocessor based laser power / energy meter (Ophir model DGX).

\section{Results and discussion}

Spectroscopy: The absorption and fluorescence emission spectra of the laser dye is shown in fig. 2. The absorption peaks are centered at $\sim 450 \mathrm{~nm}$ and $\sim 580 \mathrm{~nm}$ while the emission peak is centered at $-620 \mathrm{~nm}$.

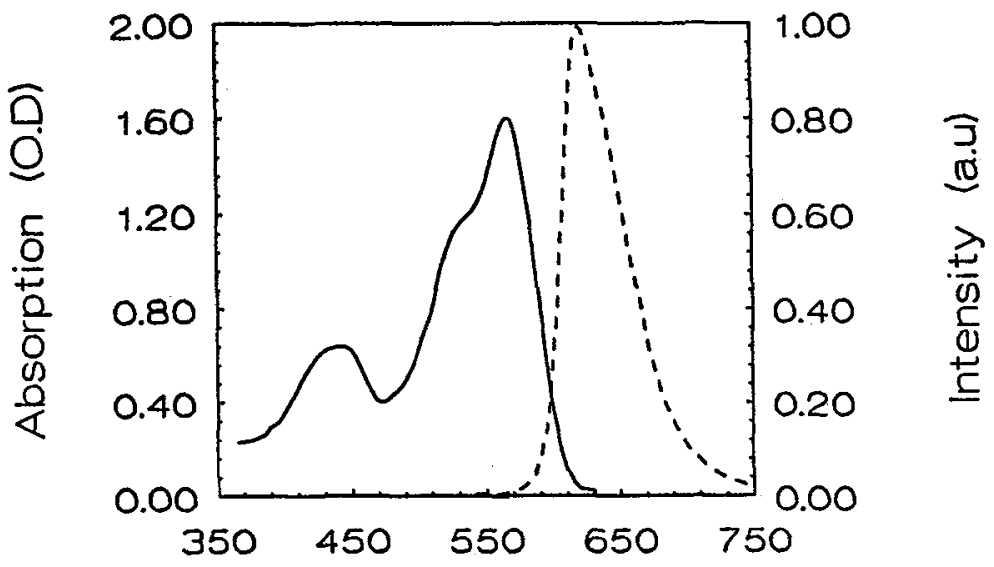

\section{Wavelength $[\mathrm{nm}]$}

Fig. 2. Absorption and emission spectra

of the dye, $1.1 \times 10 \quad M$ in composite glass.

- Absorption (left) - - - Emission (right) 
Laser: We repeatedly performed a laser action from the same sample for more than one hour each time, during which time there was no change in the laser output. The longest time during which the lasing action was performed was 3 hours - more than 500,000 pulses with no significant degradation. This high photostability behaviour of the dye was observed also by Wittwer and al. who found that the dye shows less than $10 \%$ degradation after 68 hours in $\sim 1 \mathrm{KW} / \mathrm{m}^{2}$ sun light lumination in Luminescence Solar Concentrators [12].

The output laser spectrum is presented in fig. 3. The spectrum peak was centered at about $614 \mathrm{~nm}$, with a width of about $11 \mathrm{~nm}$ FWHM (compared to $70 \mathrm{~nm}$ FWHM in the emission spectrum). The large width can be a result of the condition of the pumping - very close to threshold power and a wavelength of pumping far from the maxima absorption wavelength.

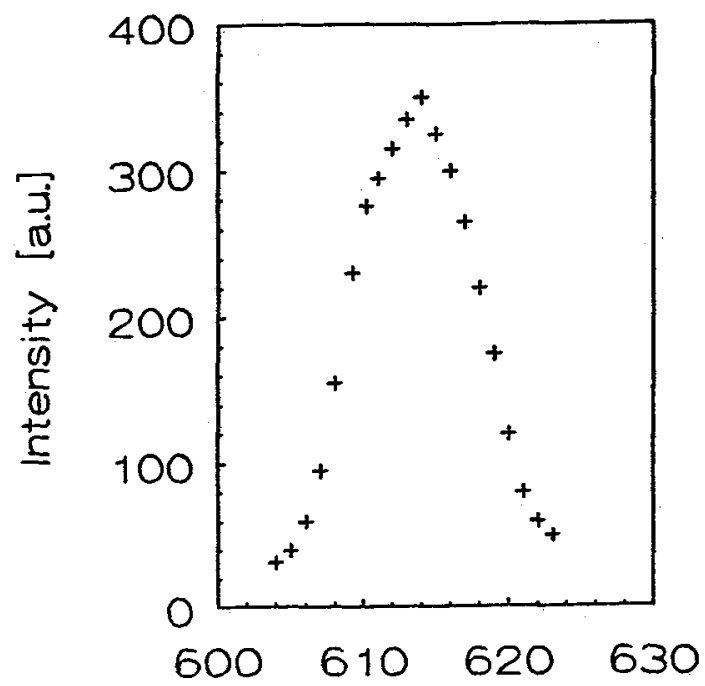

\section{Wavelength $[\mathrm{nm}]$}

Fig. 3. Output spectrum of the dye laser

for $510 \mathrm{~nm}$ pump laser, $2 \mathrm{~mJ} /$ pulse. The optical cavity

composed of $\sim 100 \%$ reflector and $\sim 50 \%$ reflecting output coupler.

The lasing threshold was measured as $1 \mathrm{~mJ} / \mathrm{pulse}$ corresponding to the power flux per pulse of $1000 \mathrm{KW} / \mathrm{cm}^{2}$. This value is higher than the calculated power flux per pulse from equation $1[13]-130 \mathrm{KW} / \mathrm{cm}^{2}$ under the same conditions.

$$
P(\lambda)=\left(N_{1} / N\right) h c / \tau_{f_{p}}^{\alpha}
$$

where $N_{1} / N$ is the minimum fraction of molecules that is raised to the first singlet state to reach the threshold of oscillation, $h$ is the Planck's constant, $c$ is the velocity of light and $\alpha_{p}$ is the absorption cross-section at the pumping wavelength (taken as: $\alpha(510)=8.3 \times 10^{-17} \mathrm{~cm}^{2}$ ). $\tau$, the fluorescent lifetime of the lasing level was calculated from equation 2 to obtained vaalue of $\tau_{f}=4.26 \mathrm{~ns}$.

$$
\eta=\tau_{f} / \tau_{r}
$$

where the radiative lifetime, $\tau_{r}$, obtained from the absorption and emission spectra using the Strickler-Berg formula [9] and the quantum eficiency taken as $\eta=0.96[10]$.

The experimental power flux is higher than the calculated because the calculated value takes into account only self-absorption losses. It should be noted that the measured threshold power flux per pulse is close to the value obtained for 
perylimide dye previously in composite glass $750 \mathrm{KW} / \mathrm{cm}^{2}(60 \mu \mathrm{J}, 8 \mathrm{~Hz}$ duration and $0.01 \mathrm{~cm}^{2}$ cross-section beam) [9].

Until now we have obtained lasing energy of only a few $\mu$ Jouls. However this can be improved through the use of a more appropriate pump laser (Nd:YAG laser operating at $532 \mathrm{~nm}$ and with energy per pulse over $100 \mathrm{~mJ} / \mathrm{pulse}$ ) and in the same time the optical quality of the sample will be improved and the dye concentration and optical cavity configuration will be optimized. In this way we expect to obtain output lasing energy up to millijouls with slope efficiency of about $20 \%$ as was observed for short-lived solid state dye laser with smiliar spectroscopic characteristics $[14,15]$.

\section{Conclusion}

A perylimide dye was impregnated into a composite silica gel glass and tested as a laser material with copper vapor laser as a source pump. The lasing action was stable for more than 500,000 pulses without changing the position of the pump laser spot. The output laser spectrum was centered at $614 \mathrm{~nm}$. By using a grating of 2400 grooves/mm as a back reflector instead of the back mirror we expect to tune the laser up to $630 \mathrm{~nm}$ in order to make it suitable for PDT by HPD.

Extended measurement of the laser charcteristics of this dye and relate dyes will be performed soon with more appropriate pump laser (Nd:YAG laser operating at $532 \mathrm{~nm}$ and with energy per pulse over $100 \mathrm{~mJ} / \mathrm{pulse}$ ). At the same time the optical quality of the sample will be improved and the dye concentration and optical cavity configuration will be optimized. Therefore, we expect to obtained improvement in the laser characteristics.

\section{Acknowledgment}

The authors thank Dr. Z. Burshtein and Dr. E. Miron for their extensive advice and to Prof. A. Lewis and his students for using his copper vapor laser and helping with its operation. One of the authors (R. Gvishi) wants to thank the Ministry of Science for the Fellowship. The work was supported by US Army European Office and the Night Vision Laboratory, Contract DAJA45-90-C-0055.

\section{References}

/1/ Cunningham, R, Lasers \& Optronics, Feb (1991) 30.

/2/ Andersson-Engels, S, Johansson, J, Svanberg, S, and Svanberg, K, Analytical Chem. 61 (1989) 1367.

/3/ Castro,D.J, Hospimedica, 4 (1990) 59.

/4/ Dougherty, T.J, Photochem. and Photobiol. 45 (1987) 879.

/5/ Andersson-Engels,S, Johansson,J, Słanberg,S, and Svanberg, K, Analytical Chem. 62 (1990) 19.

/6/ Alfano,R.R, Tata,D.B, Cordero, J, Tomashefsky,P, Longo,F.W, and Alfano, M.A, IEEE J. Quant. Elec. 20 (1984) 1567.

/7/ Reisfeld,R, and Seybold, G, Chimia 44 (1990) 295.

/8/ Reisfeld, R, Brusilovsky,D, Eyal,M, Miron,E, Burshtein, Z, and Ivri, J, Chem. Phys. Lett. 160 (1989) 43.

/9/ Reisfeld,R, Brusilovsky,D, Eyal,M, Miron,E, Burshtein, Z, and Ivri,J, Proc. Binational French - Israeli Workshop of Solid State Lasers, SPIE 1182 (1989) 230.

/10/ Seybold,G, and Wagenblast, G, Dyes and Pigments 11 (1989) 303.

11/ Reisfeld,R, Solid State Lasers Tunable in the Visible Spectrum and NonLinear Materials in Glasses. To be published in Proc. of Laser M2P Conference Grenoble, France, July, 1991.

/12/ Hinsch, A, Zastrow, A, and Wittwer,V, Solar Energy Materials 21 (1990) 151 /13/ Gvishi,R, and Reisfeld,R, J. Non-Cryst. Solids, 128 (1991) 69.

/14/ Mckiernan, J.M, Yamanka,S.A, Dunn, B, and Zink, J.I, J. Phys. Chem. 94 (1990) 5652.

15/ Salin,F, LeSaux,G, Georges,P, Brun,A, Bagnall, C, and Zarzycki,J, Optics Letters 14 (1989) 785. 